\title{
COLOCACIÓN DE UN IMPLANTE DE UN SOLO CUERPO CON TÉCNICA QUIRÚRGICA FLAPLESS, CARGA INMEDIATA Y REHABILITACIÓN EN ZIRCONIO: REPORTE DE UN CASO
}

\author{
${ }^{1}$ Martha Cecilia Mendoza Villamizar, ${ }^{2}$ Carlos Fernando Galeano Arrieta, \\ ${ }^{1}$ Odontológa U. Santo Tomás, Estudiante de II año Especialización en Rehabilitación Oral U. Santo Tomás, Bucaramanga (Colombia). \\ ${ }^{2}$ Odontólogo U. Santo Tomás, Especialista en Cirugía Maxilofacial U. El Bosque, Docente U. Santo Tomás, Bucaramanga (Colombia).
}

Autor responsable de correspondencia: Martha Cecilia Mendoza Villamizar

Correo electrónico: marthacmendo@hotmail.com

Resumen

En región de premolares con disminución de espacio en la zona edéntula, los implantes con diámetro de 3mm y de una sola pieza, son una buena alternativa, siempre que se cuente con buena calidad y densidad ósea. Acorde a la literatura, la técnica quirúrgica Flapless conserva los tejidos blandos y ofrece al paciente un postoperatorio más satisfactorio. Por su parte, la carga inmediata por estética es la más aconsejable en estos casos, además de la realización de la corona definitiva en zirconio. El propósito de estos procedimientos restaurativos fue mejorar la estética dental con tratamientos poco invasivos y mantener la función masticatoria y fonatoria normal. El reporte presenta el caso de una mujer de 48 años de edad, con espacio edéntulo del 15 reemplazado por corona en cantilever. Posterior a la realización de los procedimientos de higiene oral, se dio inicio al encerado diagnóstico, análisis en la ubicación del implante, retiro de coronas, preparación de provisionales, colocación del implante de un solo cuerpo, cirugía con técnica de Flapless, carga inmediata, y colocación de corona definitiva en zirconio (CAD-CAM). [Mendoza MC, Galeano CF. Colocación de un implante de un solo cuerpo con técnica quirúrgica Flapless, carga inmediata y rehabilitación en zirconio: reporte de un caso. Ustasalud 2012; 11: 54 - 59]

Palabras clave: Implante, Técnica Flapless, Carga inmediata, Zirconio.

\section{ONE BODY IMPLANT PLACEMENT WITH FLAPLESS SURGICAL TECHNIQUE, IMMEDIATE LOAD AND ZIRCONIUM REHAB: A CASE REPORT}

\begin{abstract}
In the premolars region with space reduction of the edentulous zone, the $3 \mathrm{~mm}$ and one piece implants are a good choice, as long as there is good quality and bone density. According to the literature found, the surgical Flapless technique preserves the soft tissues, offering the patient a more pleasant post treatment time. In these cases, the immediate esthetic load is the most recommended one, doing the definitive crown in zirconium. The purpose of these restorative procedures were to improve the dental esthetics through less invasive treatments and to maintain the normal masticatory and phonatory functions. The report shows a 48-year-old female patient, with edentulous space in the 15, replacement by a crown in cantilever. After doing the oral hygiene procedures, the wax diagnostic begin with the implant location analysis, crowns removal, temporaries collocation, one piece implant placement, technical Flapless surgery, immediate load and finally definitive zirconium crown placement (CAD-CAM).
\end{abstract}

Key words: Implant, Flapless technique, Immediate load, Zirconia.

Recibido para publicación: 15 de julio de 2011. Aceptado para publicación: 10 de marzo de 2012.

\section{INTRODUCCIÓN}

El conocimiento detallado de la estructura y la biología de los tejidos dentarios, así como el impresionante avance tecnológico traducido en nuevos instrumentos, materiales y técnicas, permite en la actualidad desarrollar procedimientos mínimamente invasivos.

La cirugía de implantes con técnica Flapless es un procedimiento predecible, siempre y cuando se realice previamente la selección de la zona. ${ }^{2}$ Aunque no se han encontrado diferencias significativas en las condiciones de ansiedad dental, el impacto en la ansiedad emocional, duración del procedimiento o dificultad técnica, el grupo de la técnica Flapless obtuvo consistentemente mayores puntajes, que el grupo del procedimiento Flap donde se reportó menor impacto en la calidad de vida y se incluyó que más pacientes reportaron menor dolor durante toda la colocación. ${ }^{3}$ Los resultados en la óseo-integración en casos donde se coloca una restauración provisional, son satisfactorios, además facilitan la formación de los contornos de la mucosa periimplantaria pues provee al paciente confort y estética. ${ }^{4}$ 
La colocación inmediata de un provisional después de la cirugía Flapless o cirugía convencional demostró para algunos pacientes de la cirugía convencional mayor edema posoperatorio y la necesidad de consumir más analgésicos. ${ }^{5}$

Hay variedad de técnicas quirúrgicas, para el desarrollo de la mucosa queratinizada alrededor de los implantes dentales. Cuando sea apropiado, estos métodos pueden aplicarse con éxito en el momento de la colocación del implante, ya que minimizan el tiempo del tratamiento, reducen la recesión del tejido marginal, proporcionan óptima salud periimplantaria, mejoran la estética y facilitan los procedimientos restaurativos. ${ }^{6}$

Los datos muestran una pérdida significativa de la cresta ósea durante los primeros seis meses de colocado el implante con diámetro de $3 \mathrm{~mm}$, seguido de un patrón estable de soporte óseo después de doce meses. La magnitud de la pérdida de masa ósea inicial es coincidente con la literatura y no se le da relevancia clínica. Se completa un alto porcentaje de las papilas, sobre todo para los incisivos laterales superiores. Este resultado puede estar relacionado con el apoyo del hueso alrededor del implante junto con los dientes adyacentes. Además, el resultado en la confección de las papilas puede ayudarse por la fabricación inmediata de las restauraciones provisionales, resultando en una mejora de la respuesta del tejido blando.?

La rehabilitación definitiva del implante fue con una corona en material biocerámico. La tecnología del zirconio ha impulsado un rápido desarrollo de la odontología libre de metales, que puede proveer una alta bio-compatibilidad, estética incrementada y mejor resistencia del material. Desde el punto de vista tecnológico, cerámicas de zirconio parcialmente estabilizada con óxido de Ytrio (Y-PSZ), también conocidas como policristales de zirconio con Ytrio tetragonal (Y-TZP), parece tener un excelente comportamiento a corto plazo; las restauraciones se procesan por maquinado suave de bloques presinterizados seguido de una sinterización a altas temperaturas. Sin embargo, no hay datos suficientes sobre la longevidad intra-oral a largo plazo y por esta razón su futuro todavía se desconoce. ${ }^{8}$

Aleatoriamente se realizó un estudio sobre 20 coronas de abutmen de zirconio y 20 de abutmen de titanio, donde se midió la profundidad de la bolsa (PPD), porcentaje de placa bacteriana (Pl), el nivel de sangrado (BOP) y la diferencia de color de la mucosa. Se produjeron dos deslaminaciones $16,7 \%$ sobre los abutment de titanio, no se encontraron diferencias significativas en las diferentes pruebas de PPD, Pl, BOP; ambas coronas las de cofias de zirconio y tita- nio inducen a un cambio en la decoloración de los tejidos blandos comparado con los tejidos blandos de los dientes naturales. ${ }^{9}$

El objetivo de este artículo fue describir una técnica quirúrgica poco traumática, en menor tiempo, que causa menor ansiedad en el paciente. El provisional se coloca de manera inmediata por lo que el paciente presenta mayor confort, restableciéndole su función y estética al iniciar desde este primer momento la conformación de tejidos blandos, alrededor del implante.

\section{REPORTE DE CASO}

\section{Características generales}

Paciente femenino de 48 años de edad que acude a las clínicas odontológicas de la Universidad Santo Tomás, Floridablanca, refiere como motivo de consulta el "cambio de coronas". En la anamnesis se determinó que era una paciente sana. Al análisis facial y craneal se encontró paciente con características meso-prosopo y meso-cefálico, ausencia de paralelismo de planos bipupilar y comisural, desarmonía de quintos, perfil recto, y al análisis de Rickets, aumento en el labio inferior a $4 \mathrm{~mm}$ (Figura 1).

En el análisis dentofacial se evidenció paciente con línea de sonrisa media, corredor bucal amplio, no coincidencia del borde incisal con el labio inferior, y biotipo periodontal delgado. En el análisis clínico presentaba restauraciones en amalgama de dientes posteriores y en premolares, obturaciones con resinas en mal estado, coronas con discrepancia (Figura 2, 3 y 4).

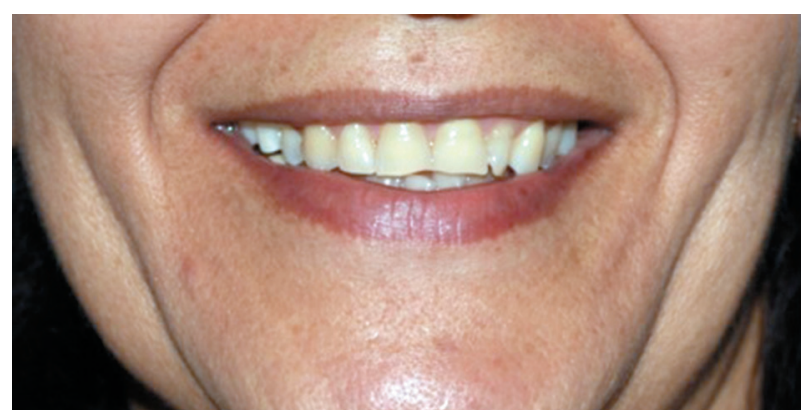

Figura 1. Fotografia inicial

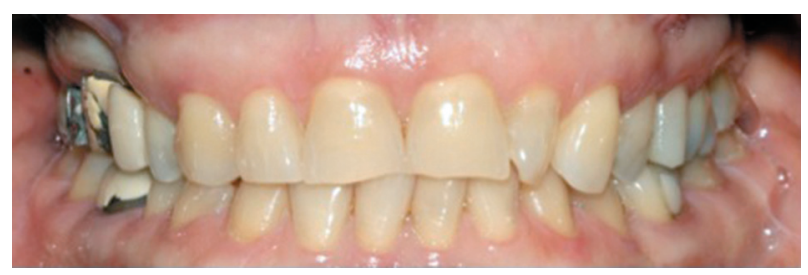

Figura 2. Vista frontal primera cita 


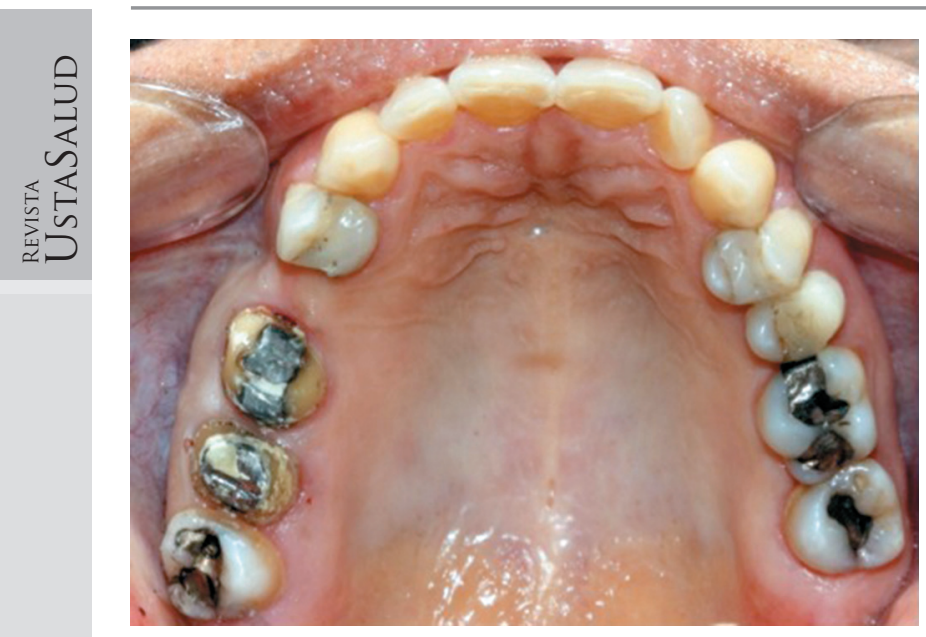

Figura 3. Vista oclusal superior

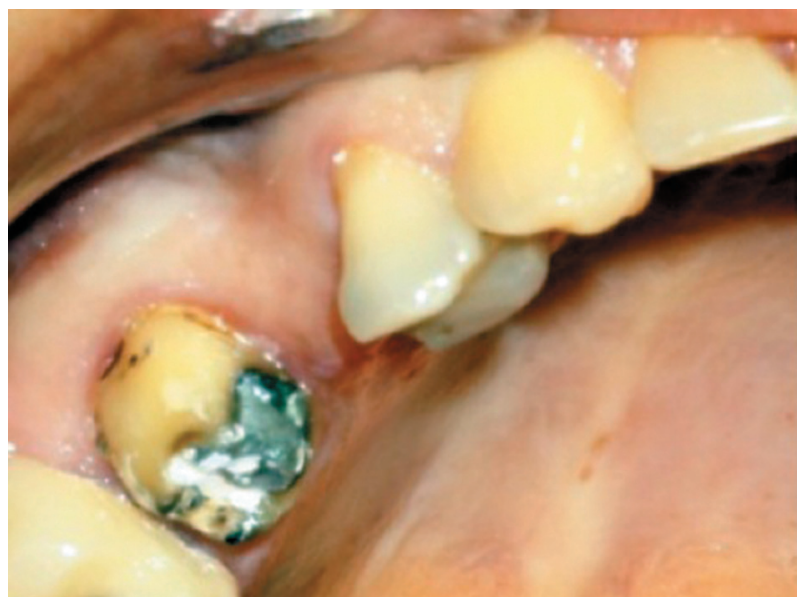

Figura 4. Espacio del diente 15

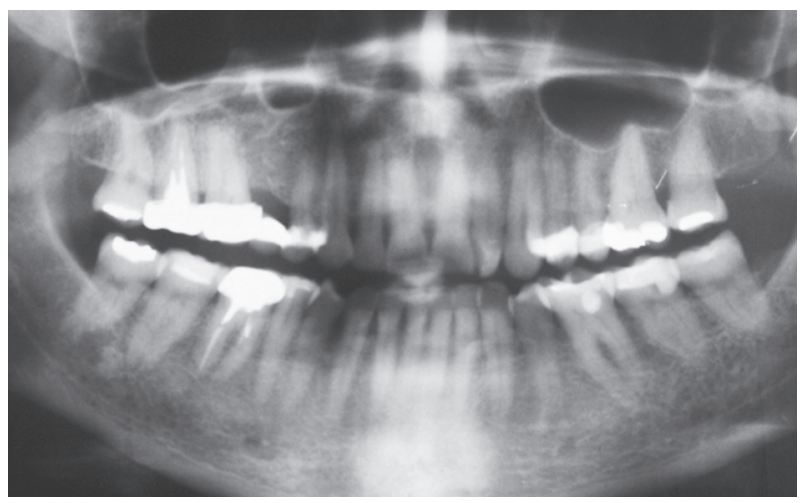

Figura 5. Radiografia panorámica

Radiográficamente, se observó a nivel del 16 zona radiopaca compatible con restauración metal cerámica, ausencia del 15 y en su reemplazo zona radiopaca compatible con restauración metal cerámica (canteliver) (Figura 5).

\section{Procedimientos}

Se realizó la fase higiénica y el raspaje coronal explicándole al paciente las técnicas de higiene oral, estos procedimientos considerados como primordiales para conservar cualquier tratamiento.

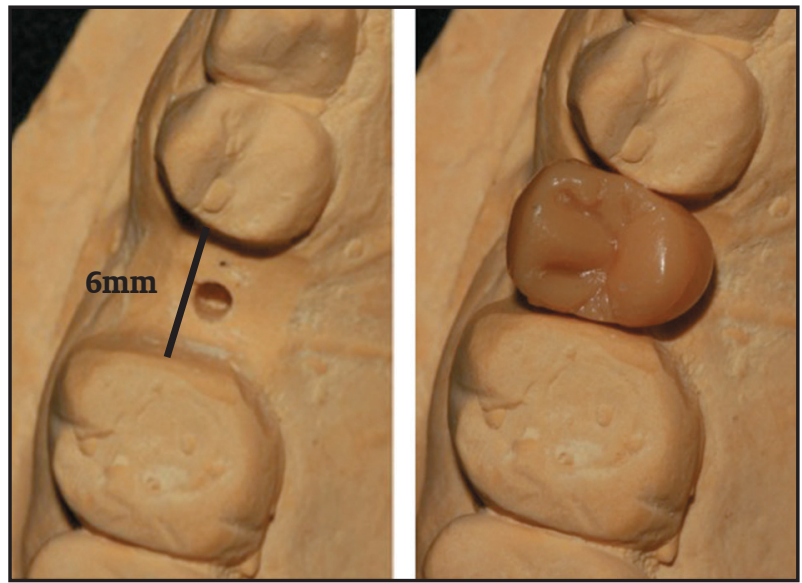

Figura 6. Encerado diagnóstico

Se realizó el encerado diagnóstico del sitio donde se colocó el implante, zona del diente 15 (Figura 6). Dada la distancia meso-distal de $6 \mathrm{~mm}$ y $8 \mathrm{~mm}$ vestíbulo-palatino, se decidió por un implante de diámetro de $3 \mathrm{~mm}$, se respetó la distancia diente-implante de $1,5 \mathrm{~mm}$ a $2 \mathrm{~mm}$ en regiones estéticas.

\section{Especificaciones del implante}

Se utilizó un implante tipo one-pice con material de aleación de Titanio - Ti-6Al-4V superficies texturizada (RBT) Diámetro: de $3.0 \mathrm{~mm}$ con una longitud de 15mm (Figura 7).

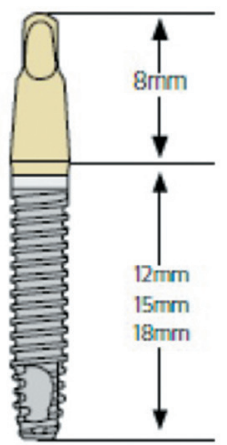

Figura 7. Implante one-piece

Fuente: www.biohorizons.com

\section{Ventajas del implante:}

- Cirugía mínima de máxima estética

- Implantes de una sola fase

- Tasa de éxito $96,7 \%$ en carga inmediata

- El tejido blando tiene menos trauma que con los típicos protocolos de dos etapas

- Permite el tratamiento de los espacios que no pueden ser manejados con implantes mayores de dos piezas

- Estos implantes disminuyen los microgap y micromovimientos 
- No se requiere tornillo pilar

- Aumenta la aposición de tejido blando.

\section{Técnica quirúrgica}

La técnica quirúrgica "Flapless" posee varias ventajas comparada con los procedimientos quirúrgicos convencionales, que incluyen la apertura de un colgajo antes de la inserción del implante (Figura 8).

El procedimiento quirúrgico consistió en la instalación del implante a través de una incisión circular de $4 \mathrm{~mm}$ de diámetro (Figura 8a), se retiró la mucosa (Figura $8 \mathrm{~b}$ y $8 \mathrm{c}$ ) y se usó la guía quirúrgica (Figura 8d) para la ubicación del implante (Figura 8e y 8f), previo estudio minucioso del caso.

\section{Ventajas de la técnica quirúrgica:}

- Excelente resultado estético

- Menor sangrado de post operatorio

- Menor incomodidad para el paciente

- Menos tiempo en la cirugía

- Menor tiempo en sanar

- Hay menos o ninguna inflamación
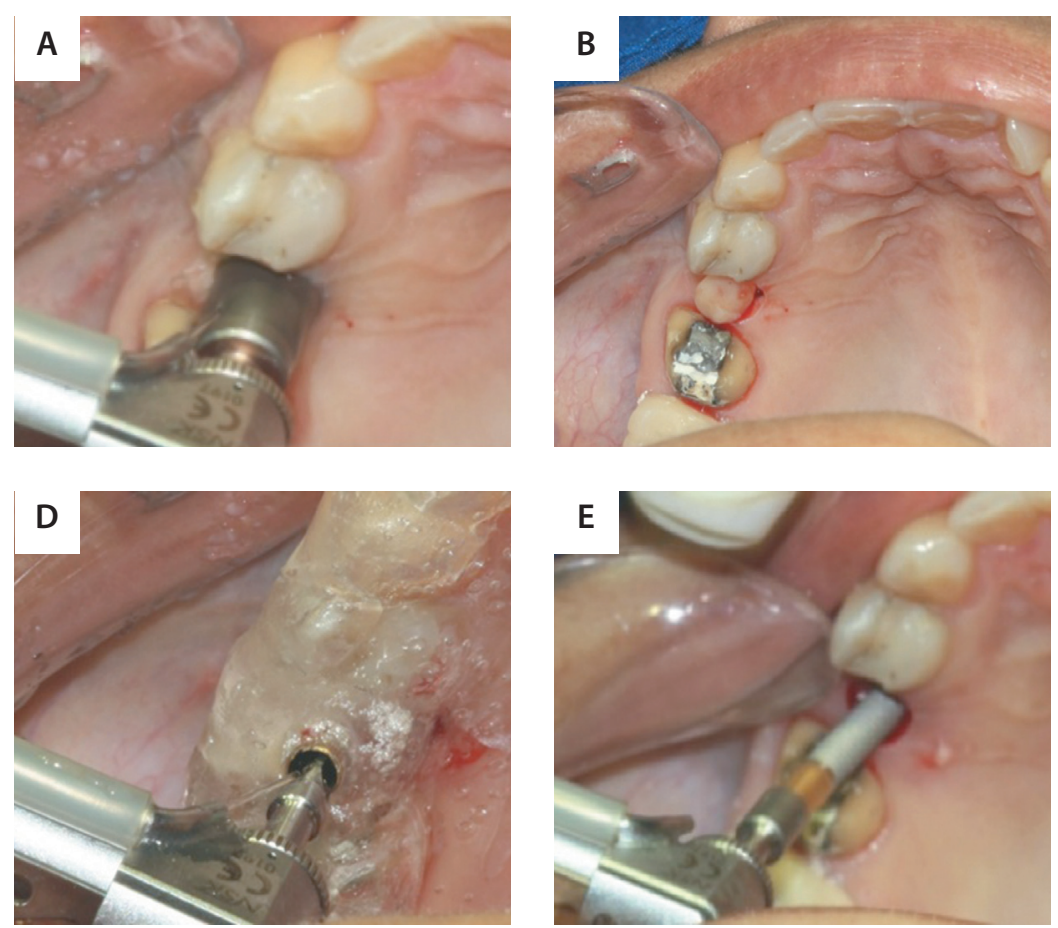
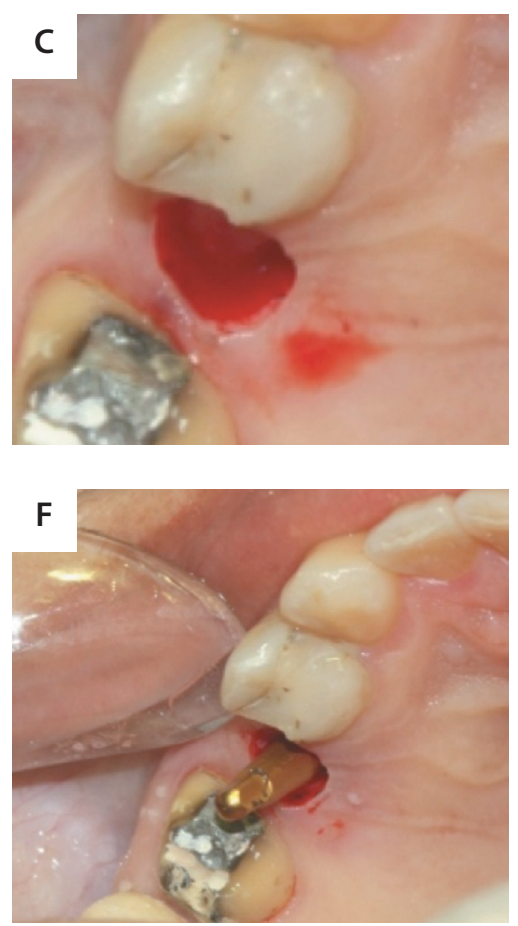

Figura 8. Secuencia quirúrgica para la colocación del implante

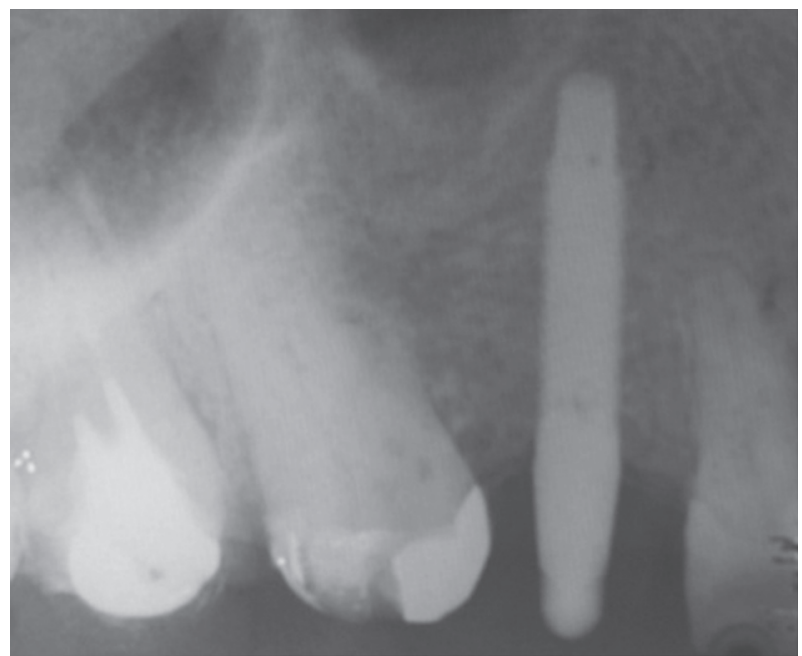

Figura 9. Radiografía post cirugía

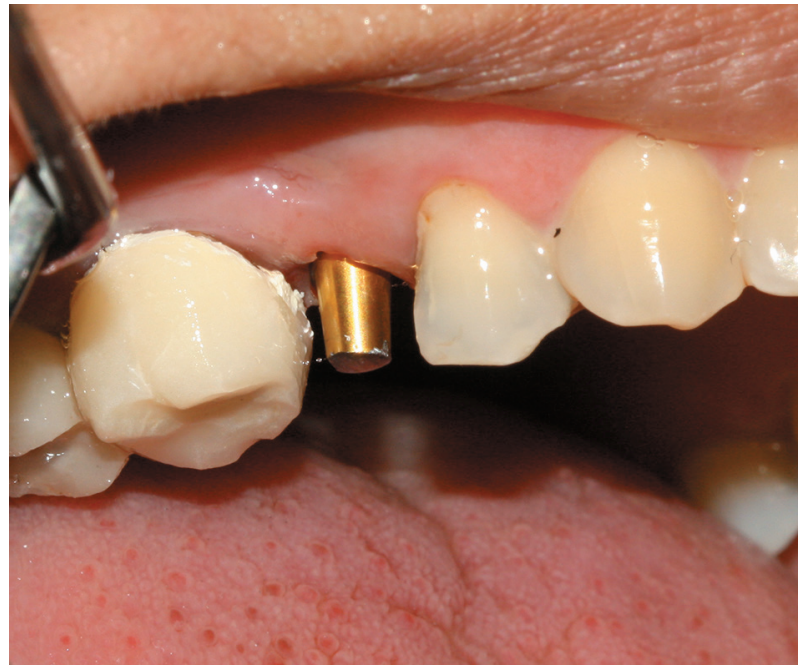

Figura 10. Arreglo del implante por oclusión 


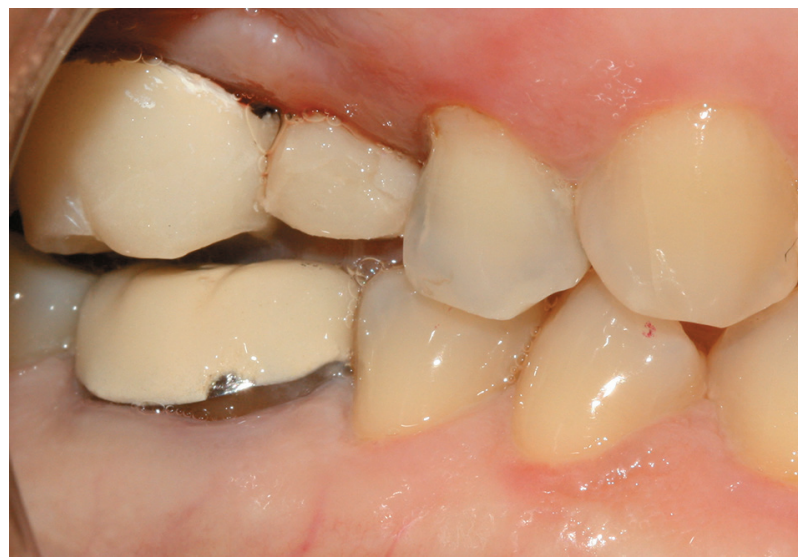

Figura 11. Provisional sobre el implante

\section{Fase de rehabilitación}

Después de la cirugía se confirmó la postura del implante mediante radiografía (Figura 9). Se observó la interferencia interoclusal haciéndose necesario disminuir la altura del implante para adecuarlo por oclusal (Figura 10). Luego se colocó un aditamento provisional, lo provee la casa fabricante o se puede confeccionar, por un periodo de tres a cuatro meses (Figura 11). Al seguir con el protocolo protésico, se tomó una impresión en silicona de adición, en un solo paso, masilla y liviana.

Con el modelo maestro y troquelado se realizó el encerado de la futura cofia. Es escaneada con el sistema Everest. Se prueba la cofia de zirconio en boca (Figura 12), radiográficamente se verificó el asentamiento de la misma (Figura13). Se envió para la colocación de la cerámica, prueba y el ajuste necesario por oclusal, se realizó la cementación definitiva (Figura 14).

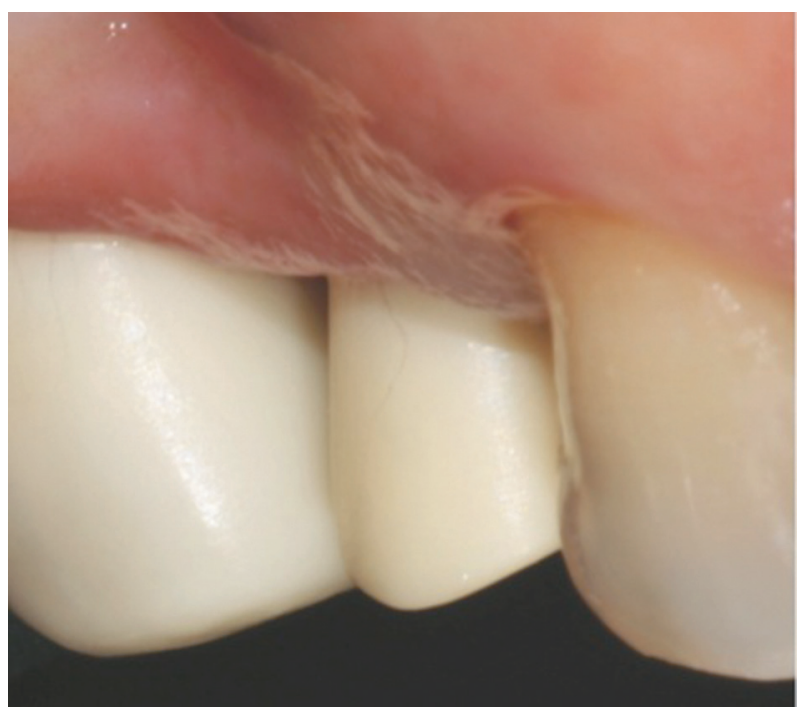

Figura 12. Cofia en zirconio

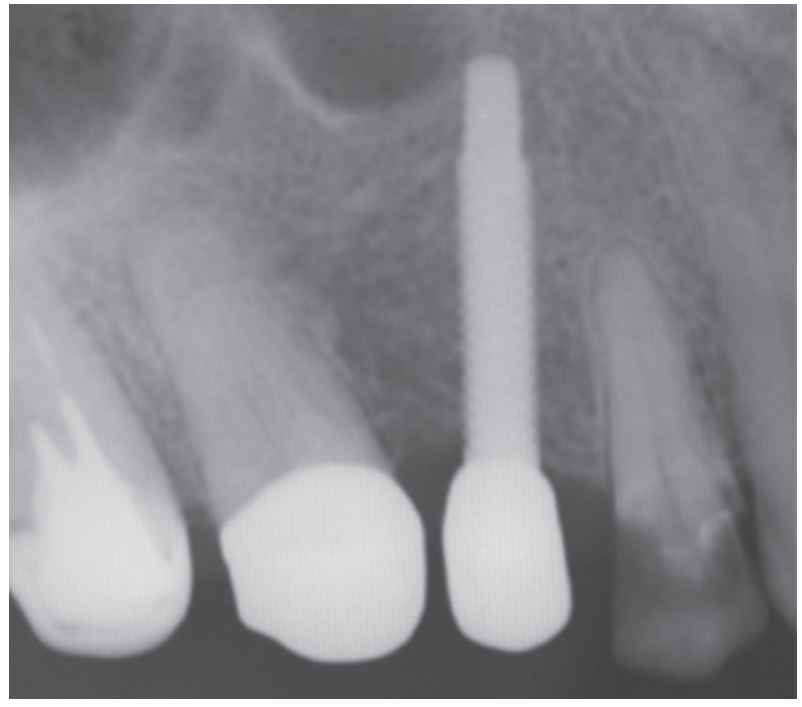

Figura 13. Radiografía prueba cofia

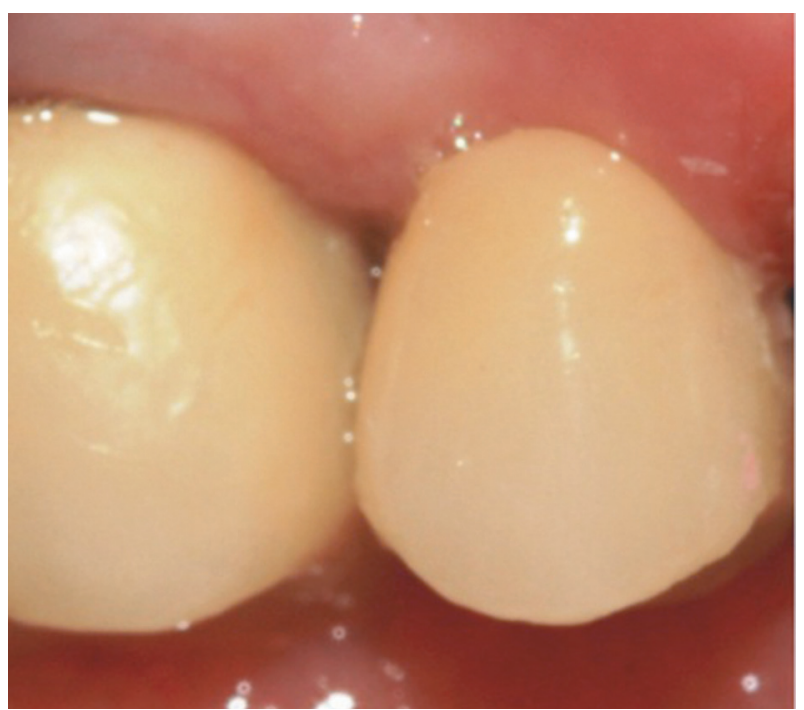

Figura 14. Corona definitiva

\section{DISCUSIÓN}

Los implantes de un solo cuerpo son una alternativa válida de tratamiento, conservadora, estética y duradera. Al analizar la influencia de los distintos parámetros en la remodelación ósea marginal después de un año de seguimiento, se encontró que los implantes colocados de un solo cuerpo, mostraron mayor remodelación ósea y preservación del tejido blando. ${ }^{10}$

Realizar un análisis cuidadoso del sitio del implante en cuanto a la altura, calidad y densidad ósea, observar la cantidad de tejido blando y tejido conectivo, para la nueva formación de las papilas. Estos implantes por su diámetro de $3 \mathrm{~mm}$ son recomendados para espacios reducidos meso-distal, como son los casos para rehabilitar laterales superiores, incisivos inferiores y premolares. 
Además de las medidas previas, es importante tener en cuenta la altura interoclusal desde la mucosa y el diente con el cual va a ocluir, para determinar la necesidad de adaptar el implante para posteriormente recibir el provisional. En la mayoría de los casos es indispensable realizar un recorte del implante para eliminar el exceso interoclusal y evitar interferencias. Al realizar alguna modificación al implante es necesario confeccionar el provisional a su nuevo diseño. Este provisional debe tener un buen punto de contacto por mesial y distal, pero en infraoclusión.

Es recomendable confirmar el encerado de las cofias de zirconio, para optimizar el proceso de las mismas. La selección apropiada de la cerámica de laminilla y del material de la estructura de zirconio, el fresado de la superficie interna de coronas durante el maquinado con CAD/CAM es el procedimiento más crítico puesto que hay áreas que concentran máximos esfuerzos tensionales y el tratamiento superficial apropiado son de extrema importancia para garantizar que las restauraciones laminadas de zirconio trabajarán de acuerdo a las demandas funcionales esperadas. Está en estudio si la deslaminación de la porcelana es directamente proporcional al grosor de la cerámica o el tratamiento de superficie de la estructura de dióxido de zirconio es el factor que influye en la deslaminación. ${ }^{11}$

\section{BIBLIOGRAFÍA}

1. Becker W, Goldstein M, Becker BE, Sennerby L. Minimally invasive flapless implant surgery: a prospective multicenter study. Clin Implant Dent Relat Res 2005; 7:21 - 27.

2. Campelo LD, Camara JR. Flapless implant surgery: A 10year clinical retrospective analysis. Int J Oral Maxillofacial Implants 2002; 17: 271 - 276.

3. Lindeboom J, van Wijk A. A comparison of two implants techniques on patient-based outcome measures: a report of flapless vs conventional flapped implant placement. Clin Oral Implants Res 2010; 21: 366 - 370.

4. Aboud M, Koeck B, Stark H. Immediate loading of singletooth implants in the posterior region. Int J Oral Maxillofacial Implants 2005; 20: 61-68.

5. Cannizzaro G, Leone M, Consolo U, Ferri V, Esposito M. Immediate functional loading of implants placed with flapless surgery versus conventional implants in partially edentulous patients: a 3-year randomized controlled clinical trial. Int J Oral Maxillofacial Implants 2008; 23: 867-875.

6. Beagle JR. Developing keratinized mucosa around nonsubmergeddental implants. Part I: The use of vascularized flaps. Perio 2005; 2: 13-22.

7. Reddy MS, O’Neal J, Haigh S, Aponte-Wesson R, Geurs NC.Initial clinical efficacy of 3-mm implants immediately placed into function in conditions of limited spacing. Int J Oral Maxillofacial Implants 2008; 23: 281-288.

8. VagkopoulouT, Koutayas SO, Koides P, Strub JR. Zirconia in dentistry: Part 1 . Discovering the nature of an upcoming bioceramic. Eur J Esthet Dent 2009; 4: 132 - 150.
9. Sailer Irena, Zembic A, Jung RE, Siegenthaler D, Holderegger C, Franz Christoph H. Randomized controlled clinical trial of customized zirconia and titanium implant abutments for canine and posterior single-tooth implant reconstructions: preliminary results at 1 year of function. Clin Oral Impl Res 2009; 20: 219-225.

10. Finne K, Rompen E, Toljanic J. Clinical evaluation of a prospective multicenter study on 1-piece implants. Part 1: Marginal bone level evaluation after 1 year of followup. Int J Oral Maxillofacial Implants 2007; 22: 226 - 234.

11. Tinschert J. Estudio clínico prospectivo de las dentaduras fijas parciales posteriores en zirconio: seguimiento a 3 años. J Prosthod 2008: 21:217 - 222.
Correos electrónicos de los autores:

Martha Cecilia Mendoza V.: marthacmendo@hotmail.com Carlos Fernando Galeano Arrieta: cfgaleanoa@gmail.com 